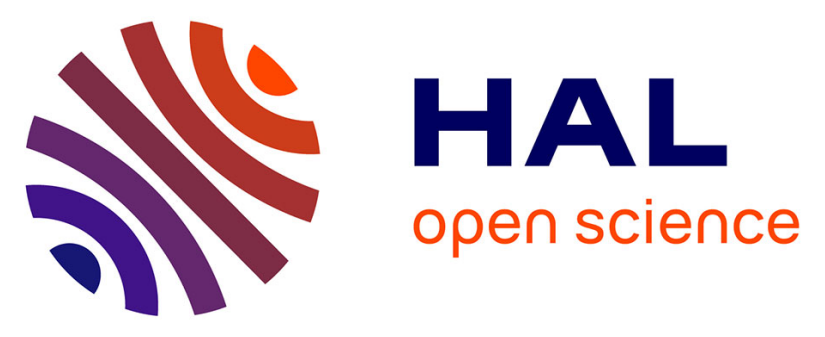

\title{
New Therapeutic Opportunities Based on DNA Mismatch Repair and BRAF Status in Metastatic Colorectal Cancer
}

Romain Cohen, Magali Svrcek, Chantal Dreyer, Pascale Cervera, Alex Duval, Marc Pocard, Jean-François Fléjou, Aimery de Gramont, Thierry André

\section{To cite this version:}

Romain Cohen, Magali Svrcek, Chantal Dreyer, Pascale Cervera, Alex Duval, et al.. New Therapeutic Opportunities Based on DNA Mismatch Repair and BRAF Status in Metastatic Colorectal Cancer. Current Oncology Reports, 2016, 18 (3), pp.18. 10.1007/s11912-016-0504-2 . hal-01310853

\section{HAL Id: hal-01310853 https://hal.sorbonne-universite.fr/hal-01310853}

Submitted on 3 May 2016

HAL is a multi-disciplinary open access archive for the deposit and dissemination of scientific research documents, whether they are published or not. The documents may come from teaching and research institutions in France or abroad, or from public or private research centers.
L'archive ouverte pluridisciplinaire HAL, est destinée au dépôt et à la diffusion de documents scientifiques de niveau recherche, publiés ou non, émanant des établissements d'enseignement et de recherche français ou étrangers, des laboratoires publics ou privés. 


\section{New therapeutic opportunities based on DNA Mismatch Repair and BRAF Status in Metastatic Colorectal Cancer}

Romain Cohen, $\mathrm{MD}^{1}$; Magali Svrcek, $\mathrm{MD}, \mathrm{PhD}^{2-3}$; Chantal Dreyer, $\mathrm{MD}^{1}$; Pascale Cervera, MD, $\mathrm{PhD}^{2-3}$; Alex Duval, $\mathrm{MD}, \mathrm{PhD}^{4}$; Marc Pocard, $\mathrm{MD}, \mathrm{PhD}^{5-6}$; Jean-François Fléjou, MD, $\mathrm{PhD}^{2-3}$; Aimery de Gramont, $\mathrm{MD}^{5,7}$; Thierry André, $\mathrm{MD}^{1,3,5}$

${ }^{1}$ Department of Medical Oncology, Hospital Saint-Antoine, APHP, 184 rue du Faubourg Saint-Antoine, Paris 75012, France

${ }^{2}$ Department of Pathology, Hospital Saint-Antoine, APHP, 184 rue du Faubourg SaintAntoine, Paris 75012, France

${ }^{3}$ University Pierre et Marie Curie (UMPC), Paris VI, 4 Place Jussieu, Paris 75005, France

${ }^{4}$ INSERM, Unité Mixte de Recherche Scientifique 938, Centre de Recherche Saint-Antoine, Equipe "Instabilité des Microsatellites et Cancers“, Equipe labellisée par la Ligue Nationale contre le Cancer, 184 rue du Faubourg Saint-Antoine, Paris 75012, France

${ }^{5}$ GERCOR, Oncology Multidisciplinary Group, 151 rue du Faubourg Saint Antoine, Paris 75011, France

${ }^{6}$ Departement of Digestive and Oncologic Surgery, Hospital Lariboisière, APHP, 2 rue Ambroise Paré, Paris 75010, France

${ }^{7}$ Department of Medical Oncology, Institut Hospitalier Franco-Britannique, 4 rue Kléber, 92300 Levallois-Perret, France 
Cohen et al., DNA Mismatch Repair and BRAF Status in Metastatic Colorectal Cancer: New therapeutic opportunities?

Correspondance: Thierry André: Department of Medical Oncology, Hospital Saint-Antoine, Assistance publique-Hôpitaux de Paris; 184, rue du Faubourg-Saint-Antoine, 75012 Paris, France; Phone: +33 (0)1 719703 87; Email: thierry.andre@aphp.fr; 
Cohen et al., DNA Mismatch Repair and BRAF Status in Metastatic Colorectal Cancer: New therapeutic opportunities?

Key Words: microsatellite instability, BRAF mutation, immune checkpoint, PD-1, PD-L1 
Cohen et al., DNA Mismatch Repair and BRAF Status in Metastatic Colorectal Cancer: New therapeutic opportunities?

\begin{abstract}
Recently, colorectal cancer (CRC) subtyping consortium identified four consensus molecular subtypes (CMS1-4). CMS1 is enriched for deficient mismatch repair (dMMR) and $B R A F^{V 600 E}$ tumors. Intriguingly, this subtype has better relapse-free survival but worse overall survival after relapse compared with the other subtypes. Growing evidence is accumulating on the benefit of specific therapeutic strategies such as immune checkpoint inhibition therapy in dMMR tumors and MAPK pathway targeted therapy in tumors harboring $B R A F^{V 600 E}$ mutation. After reviewing dMMR prognostic value, immune checkpoints as major targets for dMMR carcinomas will be highlighted. Following, $B R A F^{V 600 E}$ prognostic impact will be reviewed and therapeutic strategies with the combination of cytotoxic agents and especially the combinations of BRAF and MAPK inhibitors will be discussed.
\end{abstract}


Cohen et al., DNA Mismatch Repair and BRAF Status in Metastatic Colorectal Cancer: New therapeutic opportunities?

\section{Introduction}

$\mathrm{CRC}$ is a biologically heterogeneous disease that arises through distinct pathways including chromosomal instability (CIN), microsatellite instability (MSI), and $\mathrm{CpG}$ island methylator phenotype (CIMP). CIMP phenotype, with some degree of overlap with CIN and MSI, represents a specific type of epigenetic instability that leads to aberrant gene silencing [1]. MSI phenotype is caused by deficient DNA mismatch repair (dMMR) function resulting from an epigenetic inactivation of $M L H 1$ or from a germline mutation of one of the MMR genes (MLH1, MSH2, MSH6, and PMS2) predisposing to the Lynch' syndrome (LS).

Sporadic dMMR CRC, but not LS, is frequently associated with the v-raf murine sarcoma viral oncogenes homolog B1 $(B R A F)^{V 600 E}$ mutation. Replacement of valine by glutamic acid at position 600 within $B R A F$ gene makes mutant BRAF protein constitutively active, inducing activation of MAP kinase pathway through the phosphorylation of mitogen-activated protein kinase (MEK) downstream. Colorectal cancer subtyping consortium (CRCSC) identified four distinct consensus molecular subtypes (CMS) of CRC based on genetic and epigenetic analysis [2,3]. CMS1 includes $\mathrm{dMMR}$ and/or $B R A F^{V 600 E} \mathrm{CRC}$ and is associated with proximal location, immune activation, older age at diagnosis and female gender. CMS2 tumors exhibit high CIN, proficient MMR (pMMR), P53 mutation and/or WNT/MYC pathway; tumors with low CIN, KRAS mutation and/or phosphatidylinositol-4,5-bisphosphate 3-kinase catalytic subunit alpha (PIK3CA) mutation fall in CMS3; CMS4 tumors are characterized by transforming growth factor (TGF)-beta and/or vascular endothelial growth factor (VEGF) pathways activation and associated with mesenchymal phenotype and younger age at diagnosis. CRC with $\mathrm{dMMR}$ and/or $B R A F^{V 600 E}$ represent subtype with a poor survival after relapse (SAR) despite a favorable disease-free survival (DFS) among the four subtypes [2]. Interestingly, dMMR CRC has been recently shown as an attractive target of immunotherapy [4]. 
This review is focused on $\mathrm{dMMR}$ and $B R A F^{V 600 E}$ in metastatic CRC (mCRC). In the first part, a comprehensive overview of prognostic impact of dMMR status and of recent data of immune checkpoint modulation for dMMR mCRC is provided. In the second part, prognostic and predictive values of $B R A F^{V 600 E}$ mutation are presented followed by an update of the clinical results of targeted therapeutic strategies for $B R A F^{V 600 E}$ mCRC.

\section{Mismatch repair deficiency}

Early stage colorectal cancer

Colorectal tumors with dMMR can be detected through immunohistochemistry or polymerase chain reaction-based assay (Box 1). These tumor type is commonly associated with proximal location, high grade mucinous differentiation, and prominent lymphocyte infiltration [5]. Data show that dMMR status confers improvement DFS in patients with stage II or III CRC [6-8] and that 5-fluorouracile (5-FU) based chemotherapy is ineffective in patients exhibiting stage II dMMR tumors $[6,7,9]$. Other studies have shown that in patients with high-risk stage II and stage III tumors who received oxaliplatin-based adjuvant chemotherapy (i.e. FOLFOX), dMMR status conferred DFS benefit (André et al., in press, Journal of Clinical Oncology 2015 ) [10,11]. Interestingly, Collura et al. showed that large biallelic deletion in the T17 intron of the gene that encodes chaperone protein HSP110 sensitizes CRC to 5-FU alone or 5FU plus oxaliplatin in the adjuvant setting and affect survival of patients with stage II-III tumors $[12,13]$. Given that this mutation confers an important fraction of (about 25\%) of patients with stage II and III dMMR CRC these findings lead to reflection of the genetic features importance in chemosensitivity analysis of these tumors.

Metastatic colorectal cancer 
The impact of dMMR status on survival of patients with CRC has not been fully elucidated. The poor prevalence of $\mathrm{dMMR}$ in patients with $\mathrm{mCRC}(3 \%-5 \%)$ reinforces the low metastatic capacity of dMMR tumors and hampers the evaluation of dMMR status as a prognostic biomarker in mCRC. Indeed, the results from early studies of microsatellite instability and BRAF mutation on survival in mCRC remain inconclusive or inconsistent [14-17]. However, the pooled analysis of four phase III studies in first-line treatment of mCRC, the CAIRO, CAIRO2, COIN, and FOCUS studies, by Venderbosch et al. demonstrated that dMMR is associated with poorer overall survival (OS) (Table 1) [18]. Among 3063 patients with stage IV CRC, those who had dMMR tumors (5\%) exhibited significantly reduced progression-free survival (PFS) and OS (HR 1.33; 95\% confidence interval [CI], 1.12-1.54 and HR 1.35; 95\% CI, 1.13-1.61), respectively. Although the analysis was not sufficiently powered to test the interaction between $\mathrm{dMMR}$ and $B R A F^{V 600 E}$ status, the poor prognosis of dMMR mCRC seemed to be driven by $B R A F^{V 600 E}$ mutation (see below) [18]. The authors also suggested that the worse prognostic value observed in $B R A F^{V 600 E}$ tumors may be related to the pattern of metastatic spreading. Indeed, the low frequency of liver metastasis [17] and high rate of peritoneal disease [19] have been reported in dMMR tumors.

Therapeutic perspectives for treatment of dMMR mCRC

The immune checkpoints therapeutic strategies, such as inhibition of anti-programmed death 1 (PD-1) receptor or its ligand (PD-L1) can be considered really breakthrough agents in the targeted treatment of dMMR mCRC. A recently published phase II trial showed that patients with dMMR CRC $(N=11)$ are more responsive to anti-programmed death 1 (PD-1) antibody pembrolizumab than are pMMR CRC patients $(N=21)$ [4]. The immune-related objective response rate and the 20-week immune-related PFS rate were $40 \%$ and $78 \%$ for dMMR mCRC versus $0 \%$ and $11 \%$ for pMMR mCRC, respectively. According to immune-related 
response criteria, new lesions did not constitute disease progression if tumor burden, including new lesions, was stable or decreased. Hazard ratio for PFS was $0.10(P<0.001)$ and for OS was $0.22(P=0.05)$. Results in patients with dMMR non-CRC $(N=9)$ were similar to those with dMMR tumors. Interestingly, whole-exome sequencing analysis of dMMR and pMMR tumors revealed 20-fold more somatic mutations (inducing much more tumor-specific neoantigens) in dMMR CRC as compared with pMMR CRC $[4,20]$. These results suggested that specific immune response elements are linked to tumor genomics that is dMMR tumors may be much more responsive to checkpoint blockage with anti-PD-1 due to their incapacity to repair DNA mismatches. Higher tumor neoantigen load was associated with tumorinfiltrating lymphocytes and improved survival outcomes [21,22].

These data along with the previous observation of dense immune infiltration in those tumors support the hypothesis that dMMR CRC may be an attractive target for immunotherapy [5]. Galon and colleagues first established that the immune context as defined by the type, the density, and the location of immune cells in CRCs has the prognostic value, highlighting Thelper (Th)1-related adaptive immunity (characterized by interferon- $\gamma$ production) [23-25]. This finding was confirmed by Lal et al. on an immune gene signature which delineated dMMR CRC as a specific subgroup exhibiting high expression of Th1-related immune genes and immune checkpoint-related genes [26]. Interestingly, this active immune microenvironment is counterbalanced by the overexpression of several immune checkpoint genes including PD-1, PD-L1, cytotoxic T lymphocyte antigen 4 (CTLA-4), lymphocyte activation gene 3 (LAG-3), and indoleamine 2,3-dioxygenase (IDO) [27], making sense of immune checkpoint inhibition in dMMR CRC.

The remarkable efficacy of pembrolizumab in dMMR CRC reported in phase II study by Le et al. is an exciting discovery that opens up an entirely new field of investigations [4]. One must keep in mind that this was a very small trial where median PFS and OS for dMMR 
cohorts were not reached after a median follow-up of 36 weeks, thus larger study will be needed to confirm the findings and clarify specific issues such as effect on OS and PFS in dMMR cohort. Of note, PD-L1 expression within tumor or microenvironment was not significantly associated with either better PFS or OS. Further studies are needed to explore the PD-1 and PD-L1 expression and tumor-infiltrating immune cells as potential biomarkers of efficacy in dMMR mCRC. A phase II clinical trial of nivolumab (another anti-DP-1 antibody) and nivolumab plus ipilimumab in patients with $\mathrm{dMMR} \mathrm{mCRC}$ is ongoing (ClinicalTrials.gov Identifier: NCT02060188). Moreover, the relation between $B R A F^{V 600 E}$ status and efficacy of immune checkpoints inhibition in sporadic cancers with dMMR also warrants further exploration. Combining immunotherapy with targeted therapy in $B R A F^{V 600} \mathrm{dMMR} \mathrm{mCRC}$ may be an interesting approach which should be investigated. Finally, immune checkpoint inhibitors should be evaluated for efficacy in patients with constitutional MMR deficiency syndrome (CMMRD). CMMRD, variant of LS, is a rare condition that results from bi-allelic germline mutations of the MMR genes and is associated with a broad spectrum of childhood cancers, including CRC, hematologic malignancies, and brain tumors [28].

\section{$B R A F$-mutated colorectal cancer}

The serine/threonine-protein kinase BRAF is a downstream signaling protein in the EGFRmediated MAPK pathway that exerts its oncogenic effect through the induced phosphorylation and activation of MEK. As with RAS mutations, mutation of codon 600 within the activation segment of the kinase domain of BRAF causes constitutive activation of the MAPK pathway. Approximately $10 \%$ of CRC are BRAF-mutated, with V600E mutation in $87 \%-93 \%$ of cases. The V600E BRAF-mutated CRC are located predominantly in the right side of the colon, in older women, and typically arise from serrated adenomas and exhibit 
Cohen et al., DNA Mismatch Repair and BRAF Status in Metastatic Colorectal Cancer: New therapeutic opportunities?

high grade of differentiation. $B R A F^{V 600 E}$ mutation is strongly associated with dMMR (20\%$70 \%$ of dMMR tumors versus $5-10 \%$ of pMMR tumors) [18,29] and indicates a sporadic origin [30]. The gold standard for diagnostic analysis of a $B R A F^{V 600 E}$ mutation is currently direct sequencing. However, the clinical impact of the very rare non- $B R A F^{V 600 E}$ mutations, such as $B R A F$ codons 594 and 596 mutations, has been infrequently evaluated. The analysis of ten patients with mCRC harboring $B R A F$ codons 594 or 596 mutated tumors suggested different pathological characteristics and clinical outcomes from these of $B R A F^{V 600 E}$ tumors [31]. Longer OS was reported in patients with $B R A F$ codons 594 or 596 mutated tumors when compared with $B R A F^{V 600}$ mutated CRCs [median OS: 62.0 versus 12.6 months; hazard ratio: 0.36 (95\% confidence interval $0.20-0.64), \mathrm{P}=0.002]$.

Prognostic impact of $B R A F^{V 600 E}$ mutation in early CRC

The $B R A F^{V 600 E}$ mutation is present in $6 \%-15 \%$ [8,32-36]. Analysis of the QUASAR study, Intergroup 0-135, and MOSAIC trials data did not show any significant prognostic impact of $B R A F$ status on DFS (André et al., in press, Journal of Clinical Oncology 2015) [8,36]. In a pooled analysis of the NSABP C-07 and C-08 trials, $B R A F^{V 600 E}$ mutation was associated with poor OS (HR 1.46, $P<0.0002)$ and poor SAR (HR $2.31 P<0.0001)$ and not DFS (HR, 1.02, $P=0.86$ ) [37]. The translational study on the PETACC-3, EORTC 40993, and SAKK 60-00 trial showed that BRAF tumor V600E mutation significantly decrease OS in patients with pMMR tumors (HR 2.2, $P=0.0003$ ) [32]. Furthermore, a pooled analysis of a large cohort of 3934 patients with pMMR stage III CRC from the PETACC-8 and N0147 trials showed that $B R A F^{V 600 E}$ was an independent predictor of shorter time to recurrence, SAR, and OS [38]. These results strongly suggest that $B R A F^{V 600 E}$ mutation is associated with poor prognosis in patients with pMMR tumors. However, the positive prognostic impact of dMMR status in stage II and III CRCs seems not significantly related to the presence of $B R A F^{V 600 E}$ mutation. 
Cohen et al., DNA Mismatch Repair and BRAF Status in Metastatic Colorectal Cancer: New therapeutic opportunities?

The strong inter-relation between $B R A F^{V 600 E}$ mutation and dMMR status is particularly interesting given that these two biomarkers exhibit opposite prognostic effects in mCRC. Thus dMMR status may reduce the risk of recurrence induced by $B R A F^{V 600 E}$ CRCs, but its positive prognostic value may be eclipsed by the impact of $B R A F^{V 600 E}$ after relapse, explaining the association of $B R A F^{V 600 E}$ mutation with SAR and OS but not with DFS.

Prognostic impact of $\mathrm{BRAF}^{V 600 E}$ mutation in $\mathrm{mCRC}$

$B R A F^{V 600 E}$ is associated with a higher frequency of peritoneal and distant lymph node metastases and a decreased lower rate of lung metastases compared to BRAF wild-type tumors $\left(B R A F^{W T}\right)[17,19]$. This distinct pattern of metastatic spread may be a possible explanation for the poor prognostic impact of $B R A F^{V 600 E}$ in mCRC. Indeed, the analysis of the NSABP C-07 and C-08 trials reported by Gavin et al. clearly showed that BRAF V600E was associated with poor prognosis after relapse in stage II and III CRC [37]. In a pooled analysis of the CAIRO, CAIRO-2, COIN, and FOCUS trials, prevalence of $B R A F^{V 600 E}$ was $8.2 \%$ in mCRC [18]. Median PFS and OS were significantly reduced for patients with $B R A F^{V 600 E}$ compared with $B R A F^{W T}$ tumors (HR 1.34, $P<0.001$ versus HR 1.91, $P<0.001$ ). In pMMR mCRC stratified by $B R A F^{V 600 E}$ status, the median OS was significantly decreased in patients with $B R A F^{V 600 E}$ compared to those with $B R A F^{W T}$ tumors (11.3 months versus 17.3 months; HR 1.94, $P<0.001)$. In dMMR mCRC, no statistically significant difference was observed for OS between two groups of patients (11.7 months in $B R A F^{V 600 E}$ versus 15 months in $B R A F^{W T}$ tumors; HR 1.51, 95\% CI 0.93-2.46). But in patients with $B R A F^{V 600 E} \mathrm{mCRC}$ OS was poor, regardless of their dMMR status (HR 1.05, 95\% CI 0.68-1.63). In $B R A F^{W T} \mathrm{mCRC}$, the median OS was no significantly different between patients with dMMR and pMMR tumors (HR 1.22; 95\% CI 0.91-1.65). Thus, pejorative prognostic impact of dMMR status in mCRC seems to be driven by the $B R A F^{V 600 E}$ status. 
Cohen et al., DNA Mismatch Repair and BRAF Status in Metastatic Colorectal Cancer: New therapeutic opportunities?

The prevalence of $B R A F^{V 600 E}$ in dMMR mCRC observed by Venderbosch (a pooled analysis of the CAIRO, CAIRO2, COIN, and FOCUS studies) [18] was higher than that reported in early-stage dMMR CRC (34.6\% versus $24 \%$ in the PETACC-3 trial, $30 \%$ in the QUASAR trial) $[8,32]$. The observed increase of prevalence between localized and advanced dMMR CRC reinforces $B R A F^{\mathrm{V} 600 \mathrm{E}}$ mutation as a significant poor prognostic factor in dMMR tumors.

Predictive impact of $B R A F^{V 600 E}$ status in mCRC

Constitutively active $B R A F^{V 600 E}$ mutations are almost always mutually exclusive of $K R A S$ mutations. The activating mutations in $K R A S$ induce constitutive Ras/MAPK signaling, which cannot be suppressed by epidermal growth factor receptor (EGFR) inhibition. It has been suggested that $B R A F^{V 600 E}$ may be predictive of resistance to anti- EGFR monoclonal antibody therapies, although this association remains controversial, but its negative predictive prognostic value has been established. The analysis of patients with $B R A F^{V 600 E} \mathrm{mCRC}$ of the CRYSTAL phase III trial showed no significant difference, but a trends of better PFS or OS between patients treated with FOLFIRI alone and FOLRIRI plus cetuximab in first-line setting (HR 0.93, 95\% CI 0.42-2.06 and HR 0.91, 95\% CI 0.51-1.62) [39]. Similar results were observed in the PRIME and FIRE-3 phase III trials [40-42]. Anti-EGFR monoclonal antibody survival benefit was observed in patients with $B R A F^{V 600 E}$ tumors (Table 2). In pretreated $B R A F^{V 600 E}$ mCRC, $B R A F^{V 600 E}$ status did not discriminate between responders and nonresponders to anti-EGFR therapy [43-47]. In addition, there is insufficient evidence to conclude that patients mCRC do not benefit from anti-EGFR monoclonal antibody in the presence of $B R A F^{V 600 E}$ as shown in the meta-analysis of randomized controlled trials of CRC patients reported by Rowland and colleagues [48]. Based on these findings, there is yet no sufficient data that would justify the exclusion of anti-EGFR monoclonal antibody therapy for patients with $R A S^{W T} / B R A F^{V 600 E} \mathrm{mCRC}$. 
Cohen et al., DNA Mismatch Repair and BRAF Status in Metastatic Colorectal Cancer: New therapeutic opportunities?

Targeting $B R A F^{V 600 E} \mathrm{mCRC}$

Considering that patients with $B R A F^{V 600 E} \mathrm{mCRC}$ have a lower probability of receiving further lines of chemotherapy because of their poor prognosis [49], further intensification of standard therapy may be a pragmatic and an efficient therapeutic approach to overcome this issue. The intensification of the treatment with the triplet regimen FOLFOXIRI (5-FU, leucovorin (LV), oxaliplatin, and irinotecan) in combination with bevacizumab has been evaluated for chemotherapy-naive CRC patients in phase II and III studies with encouraging results [5054]. In the phase II study by Masi et al. median PFS was 12.8 months and OS was 23.8 months for FOLFOXIRI/bevacizumab in $B R A F^{V 600 E}$ mutated patients $(n=10)$ [50]. The addition of bevacizumab to FOLFOXIRI regimen increased treatment efficacy in the a pooled analysis of $25 B R A F^{V 600 E}$ patients reported by Salvatore and colleagues [51]. At a median follow-up of 34.1 months, the pooled set of patients showed a median PFS of 11.8 months and a median OS of 23.8 months. In the phase III TRIBE trial patients were randomized either to FOLFOXIRI/bevacizumab or FOLFIRI/bevacizumab. In this study, patients with $B R A F^{V 600 E} \mathrm{mCRC}$ showed a non-significant increase of OS when treated with FOLFOXIRI/bevacizumab (19 months versus 10.7 months; HR 0.54, 95\% CI 0.24-1.20). FOLFOXIRI/bevacizumab treatment was tolerable with $8.8 \%$ febrile neutropenia and $18.8 \%$ grade 3-4 diarrhea [52-54]. These data suggest that FOLFOXIRI plus bevacizumab is a serious and valid option for the first-line treatment of chemotherapy-naive fit patients with $B R A F^{V 600 E} \mathrm{mCRC}$.

Vemurafenib, an oral inhibitor of the mutant BRAF kinase achieved minimal clinical activity in $B R A F^{V 600 E}$ advanced melanoma patients, showing a significant benefit in response rates, PFS, and OS [55,56]. However, vemurafenib alone achieved minimal clinical activity in $B R A F^{V 600 E}$ mCRC patients [57]. Prahallad and colleagues demonstrated that BRAF V600E inhibition causes a rapid feedback activation of EGFR in CRC cell lines [58]. Based on this 
observation, the combination of vemurafenib with an anti-EGFR therapy or a MEK inhibitor may be more effective way to overpass BRAF inhibitor primary resistance in $B R A F^{V 600 E}$ mCRC in the clinical setting. Two recent studies showed that the combination of a BRAF inhibitor dabrafenib and a MEK inhibitor trametinib significantly improved OS in patients with $B R A F^{V 600 E}$ metastatic melanoma. The combination significantly improved OS compared with single-agent inhibition $[59,60]$. Moreover, dual BRAF-MEK inhibition by dabrafenib and trametinib was shown to reduce the single-agent cutaneous toxicities probably due to their opposing effects on cellular functions and signaling. In cells expressing $B R A F^{W T}$ such as epidermal keratinocytes, BRAF inhibitors binding to one member of RAF homo/heterodimers inhibit one promoter, but paradoxally transactivate the drug-free protomer, which results in increased downstream the RAF-MEK-ERK signaling, while the combination of EGFR and MEK inhibitors effectively block the MAPK signaling pathway [61,62]. Several drug combinations are currently tested in $B R A F^{V 600 E} \operatorname{mCRC}$ (Table 3). Combined inhibition with dabrafenib and trametinib showed limited activity in $B R A F^{V 600 E}$ stage IV mCRC with a response rate of $12 \%$, including one prolonged complete response (> 22 months) in phase I/II trial [63]. The further logical next step for treatment optimization was the "horizontal" and "vertical" inhibition of MAPK pathway with the triplet therapy including dabrafenib, trametinib, and panitumumab [64]. Atreya et al. conducted phase I/II trial of this combination in BRAF mutated mCRC. Overall, $11 \%, 36 \%$, and $54 \%$ of patients treated with the combination of panitumumab and dabrafenib, the triple regimen, and the combination of trametinib plus panitumumab, respectively, required dose reductions or interruptions. The greatest proportion of serious dermatologic toxicities with the combination of panitumumab and trametinib without the BRAF inhibitor were reported, thus that addition of dabrafenib to the doublet lessened skin-related toxicity. Overall, $26 \%$ of confirmed response rates with one confirmed complete response were observed [64]. The triplet therapy therefore appears to be 
Cohen et al., DNA Mismatch Repair and BRAF Status in Metastatic Colorectal Cancer: New therapeutic opportunities?

more active in $B R A F^{V 600 E} \mathrm{mCRC}$ than the double combination of BRAF and MEK inhibitors, but is associated with significant skin toxicities. The combination of panitumumab with vemurafenib for $B R A F^{V 600 E} \mathrm{mCRC}$ has shown interesting hints of enhanced clinical activity in a pilot trial by Yaeger et al. [65]. Two of 12 evaluable patients (13\%) had confirmed longlasting partial responses. The combination was well tolerated with less cutaneous toxicity than expected with either agent. The activity of the triplet combination of vemurafenib with cetuximab and irinotecan for the treatment of 19 BRAF-mutated mCRC was evaluated in a phase Ib trial [66]. The majority of patients (74\%) had received prior irinotecan and nearly half had prior exposure to cetuximab. Confirmed response rate was 53\% and median PFS was 7.7 months. These results form the basis for the ongoing phase 2 Intergroup Study S1402 trial of irinotecan and cetuximab with or without vemurafenib in cetuximab-naive $B R A F^{V 600 E}$ patients (ClinicalTrials.gov Identifier: NCT02164916). MO29112 is an ongoing phase II trial evaluating a biomarker-driven maintenance strategy for FOLFOX plus bevacizumab first-line therapy in patients with $B R A F^{V 600 E}$ mCRC (ClinicalTrials.gov Identifier: NCT02291289). Patients with $B R A F^{V 600 E} \mathrm{mCRC}$ without progressive disease after induction with the FOLFOX plus bevacizumab combination are randomized to receive either 5-FU/LV with cetuximab and vemurafenib or fluoropyrimidine (5-FU/LV or capecitabine) plus bevacizumab. Patients without targetable tumor biomarker will receive fluoropyrimidine and bevacizumab with or without MPDL3280A (anti-PD-L1 antibody).

Considering that poor prognosis conferred by $B R A F^{V 600 E}$, clinical trial enrollment should be systematically considered and planned so that patients with tumors harboring this mutation have the opportunity to receive these innovative-targeted therapies.

\section{Conclusion}


Given the high frequency of CRC worldwide, $\mathrm{dMMR}$ and $B R A F^{V 600 E} \mathrm{CRC}$ still constitutes meaningful group of patients ( 9 to $11 \%$ of patients with mCRC). New therapeutic strategies are urgently needed for those patients. $\mathrm{dMMR}$ and $B R A F^{V 600 E}$ are closely interlinked, with specific prognostic and predictive values for both of them. Considering the metastatic setting, new horizons have been opened in the field of dMMR mCRC through immune checkpoint inhibitors, which may be probably the next revolution for patients with dMMR carcinomas. Further studies will have to confirm these attractive results and investigate biological mechanisms underlying sensitivity of dMMR carcinomas to immunotherapy. Dramatic prognosis conferred by $B R A F^{V 600 E}$ mutation stresses the urge for new therapeutic strategies. Targeting $B R A F^{V 600 E}$ appears challenging in the context of mCRC and combinations of BRAF inhibitors with other MAPK inhibitors and cytotoxic agents have to be evaluated. For these reasons, patients with $\mathrm{dMMR}$ or $B R A F^{V 600 E} \mathrm{mCRC}$ have to be systematically identified because of the potential innovative therapeutic opportunities offered in the ongoing clinical trials. 


\section{$\underline{\text { Boxes }}$}

Box 1: How to determine dMMR status?

DNA mismatch repair (MMR) machinery consists of a complex of proteins MutL homolog 1 (MLH1), MutS homolog 2/ (MSH2/), and postmeiotic segregation increased 2 (PMS2), which form heterodimers MutL $\alpha$ (MLH1-PMS2) and MutS $\alpha$ (MSH2-MSH6). Mutations of the MMR genes result in loss of expression of the corresponding protein and may be detected by immunohistochemistry (IHC). Considering the predominance of $M L H 1$ and $M S H 2$ gene mutations in Lynch syndrome and the epigenetic inactivation of $M L H 1$ in sporadic dMMR colorectal cancers (CRC), IHC with anti-MLH1 and anti-MSH2 antibodies can be used to detect MMR-deficient (dMMR) tumors [67]. MLH1 and MSH2-negative tumors are systematically associated with loss of MLH1/PMS2 and MSH2/MSH6 function (MSH6 unstable in the absence of MSH2), respectively [68]. Isolated absence of MSH6 or PMS2 protein do not always produce loss of their corresponding partner, however these are rare cases. A potential drawback to IHC is that it may fail to detect dMMR cases with missense mutations, which not always correlate with loss of protein expression. Nevertheless, MMR IHC analysis is as effective for detecting dMMR CRC as microsatellite genotyping [69]. MMR deficiency results in microsatellite instability (MSI). Microsatellites are highly repetitive DNA sequences of one to six nucleotides distributed throughout the genome, which are frequently copied incorrectly. The MMR system is responsible for their detection and correction. Genotyping microsatellites through polymerase chain reaction (PCR)-based assay is the standard method to detect MSI. First guidelines on MSI analysis recommended a reference panel (Bethesda panel) of five microsatellites comprising two mononucleotide repeats (BAT-26 and BAT-25), and three dinucleotide repeats (D2S123, D5S346, and D17S250) [70]. This method is comparing the differences in allelic sizes obtained from tumor 
Cohen et al., DNA Mismatch Repair and BRAF Status in Metastatic Colorectal Cancer: New therapeutic opportunities?

and normal DNA; MSI being defined by instability for at least two of the five microsatellites. However, the Bethesda panel has several limitations, mainly due to the difficulty to interpret PCR amplification of dinucleotide markers. Therefore, the revised Bethesda guidelines recommend the use of mononucleotide repeats instead of dinucleotide repeats [71]. An alternative pentaplex-PCR assay comprising five quasi-monomorphic mononucleotide repeats (BAT-26, BAT-25, NR-21, NR-24, and NR-27) has been developed; MSI status being defined by the instability of three microsatellites or more. This optimized pentaplex PCR assay (Pentaplex Promega $\odot$ ) is at least as sensitive and specific for detection of MSI status as the Bethesda panel and obviate the need for normal matching DNA for comparison [67]. 
Cohen et al., DNA Mismatch Repair and BRAF Status in Metastatic Colorectal Cancer: New therapeutic opportunities?

\section{$\underline{\text { Tables }}$}

Table 1 Prognostic value of deficient mismatch repair in metastatic colorectal cancer

\begin{tabular}{llccc}
\hline Author & No. of patients & \multicolumn{1}{c}{ OS } & P-value \\
& (dMMR/pMMR) & \multicolumn{2}{c}{ (months) } & \\
& & dMMR & pMMR & \\
\hline Brueckl et al. [14] & $43(7 / 36)$ & 33 & 19 & 0.021 \\
Des Guetz et al. [15] & $40(9 / 31)$ & 16 & 22.5 & 0.16 \\
Koopman et al. [16] & $515(18 / 497)$ & 10.2 & 17.9 & 0.41 \\
Tran et al. [17] & $350(40 / 310)$ & 11.1 & 22.1 & 0.001 \\
Venderbosch et al. [18] & $3063(153 / 2910)$ & 13.6 & 16.8 & 0.001 \\
\hline
\end{tabular}


Cohen et al., DNA Mismatch Repair and BRAF Status in Metastatic Colorectal Cancer: New therapeutic opportunities?

Table $2 B R A F^{V 600 E}$ mutation predictive value for anti-EGFR monoclonal antibody therapy

\begin{tabular}{|c|c|c|c|c|c|}
\hline Trial & & & PFS & & OS \\
\hline & patients & $\begin{array}{l}\text { Median } \\
\text { (months) }\end{array}$ & $\begin{array}{l}\text { Hazard ratio } \\
\qquad 95 \% \mathrm{CI}] \\
P \text {-value }\end{array}$ & $\begin{array}{l}\text { Median } \\
\text { (months) }\end{array}$ & $\begin{array}{c}\text { Hazard ratio } \\
\text { [95\% CI]; } \\
P \text {-value }\end{array}$ \\
\hline CRYSTAL [39] & 59 & & & & \\
\hline FOLFIRI & 33 & 5.6 & & 10.3 & \\
\hline FOLFIRI + cetuximab & 26 & 8.0 & $\begin{array}{c}0.93[0.42-2.06] \\
0.87\end{array}$ & 14.1 & $\begin{array}{c}0.91[0.51-1.62] \\
0.74\end{array}$ \\
\hline PRIME [40] & 53 & & & & \\
\hline FOLFOX & 29 & 5.4 & & 9.2 & \\
\hline FOLFOX + panitumumab & 24 & 6.1 & $\begin{array}{c}0.58[0.29-1.15] \\
0.12\end{array}$ & 10.5 & $\begin{array}{c}0.90[0.46-1.76] \\
0.76\end{array}$ \\
\hline FIRE-3 [42] & 48 & & & & \\
\hline FOLFIRI + bevacizumab & - & 6.0 & & 13.7 & \\
\hline FOLFIRI + cetuximab & - & 4.9 & $\begin{array}{c}0.87[0.49-1.57] \\
0.65\end{array}$ & 12.3 & $\begin{array}{c}0.87[0.47-1.61] \\
0.65\end{array}$ \\
\hline
\end{tabular}


Cohen et al., DNA Mismatch Repair and BRAF Status in Metastatic Colorectal Cancer: New therapeutic opportunities?

Table 3 Targeted therapies in clinical development for $B R A F^{V 600 E}$ metastatic colorectal cancer

\begin{tabular}{lcccccc}
\hline Treatment & $\begin{array}{c}\text { Clinicaltrial.gov } \\
\text { Identifier }\end{array}$ & Phase & $\begin{array}{c}\text { No. of } \\
\text { patients }\end{array}$ & $\begin{array}{c}\text { OR } \\
\text { (\%) }\end{array}$ & $\begin{array}{c}\text { (\%) } \\
\text { (months) }\end{array}$ & $\begin{array}{c}\text { PFS } \\
\text { (m) }\end{array}$ \\
\hline Vemurafenib [57] & NCT00405587 & I & 19 & 5 & 21 & 3.7 \\
Vemurafenib [72] & NCT01524978 & II & 10 & 0 & - & - \\
Vemurafenib + panitumumab [65] & NCT01791309 & I/II & 15 & 13 & 53 & 3.2 \\
Vemurafenib + cetuximab [72] & NCT01524978 & II & 11 & - & 36 & - \\
Vemurafenib + cetuximab + CPT11 [66] & NCT01787500 & Ib & 19 & 35 & - & 7.7 \\
Dabrafenib + trametinib [63] & NCT01750918 & I/II & 43 & 12 & 51 & 3.5 \\
Dabrafenib + panitumumab [64] & NCT01750918 & I/II & 20 & 10 & 80 & 3.4 \\
Dabrafenib + trametinib + panitumumab & NCT01750918 & I/II & 35 & 26 & 60 & 4.1 \\
[64] & & & & & & 4 \\
Encorafenib [73] & NCT01436656 & I & 18 & 0 & 67 & 4 \\
Encorafenib + cetuximab [74] & NCT01719380 & I & 26 & 23 & - & 3.7 \\
Encorafenib + cetuximab + BYL719 [74] & NCT01719380 & I & 28 & 32 & - & 4.3 \\
\hline
\end{tabular}

OR: objective response; SD : stable disease; PFS: progression-free survival 
Cohen et al., DNA Mismatch Repair and BRAF Status in Metastatic Colorectal Cancer: New therapeutic opportunities?

\section{Acknowledgments:}

Authors would like to thank Magdalena Benetkiewicz for reviewing/editorial assistance.

\section{Conflicts of interest:}

TA is principal investigator for studies in metastatic colorectal cancer sponsored by Bristol Mayer Squib, Novartis, and Roche. TA received honoraria or advisory board consultant fees from Roche and Bristol Mayer Squib. AdG received honoraria for advisory from Roche.

\section{Funding:}

None 


\section{References}

Papers of particular interest, published recently, have been highlighted as:

•- Of importance

-. Of outstanding importance

1. Jass JR. Classification of colorectal cancer based on correlation of clinical, morphological and molecular features. Histopathology. 2007;50:113-30.

2. Dienstmann R, Guinney J, Delorenzi M, Reynies AD, Roepman P, Sadanandam A, et al. Colorectal Cancer Subtyping Consortium (CRCSC) identification of a consensus of molecular subtypes. Journal of Clinical Oncology. 2014;32:5s (suppl; abstr 3511).

3. Marisa L, de Reyniès A, Duval A, Selves J, Gaub MP, Vescovo L, et al. Gene Expression Classification of Colon Cancer into Molecular Subtypes: Characterization, Validation, and Prognostic Value. Kemp C, editor. PLoS Medicine. 2013;10:e1001453.

\section{4. $\bullet-M M R$ deficiency predicts efficacy of immune checkpoint inhibition with PD-1 inhibitor} pembrolizumab in colorectal and non-colorectal carcinomas.

5. Dolcetti R, Viel A, Doglioni C, Russo A, Guidoboni M, Capozzi E, et al. High prevalence of activated intraepithelial cytotoxic $\mathrm{T}$ lymphocytes and increased neoplastic cell apoptosis in colorectal carcinomas with microsatellite instability. The American journal of pathology. 1999;154:1805-13.

6. Ribic CM, Sargent DJ, Moore MJ, Thibodeau SN, French AJ, Goldberg RM, et al. Tumor microsatellite-instability status as a predictor of benefit from fluorouracil-based adjuvant chemotherapy for colon cancer. New England Journal of Medicine. 2003;349:247-57.

7. Sargent DJ, Marsoni S, Monges G, Thibodeau SN, Labianca R, Hamilton SR, et al. Defective Mismatch Repair As a Predictive Marker for Lack of Efficacy of Fluorouracil-Based Adjuvant Therapy in Colon Cancer. Journal of Clinical Oncology. 2010;28:3219-26.

8. Hutchins G, Southward K, Handley K, Magill L, Beaumont C, Stahlschmidt J, et al. Value of Mismatch Repair, KRAS, and BRAF Mutations in Predicting Recurrence and Benefits From Chemotherapy in Colorectal Cancer. Journal of Clinical Oncology. 2011;29:1261-70.

9. Sinicrope FA, Foster NR, Thibodeau SN, Marsoni S, Monges G, Labianca R, et al. DNA Mismatch Repair Status and Colon Cancer Recurrence and Survival in Clinical Trials of 5-Fluorouracil-Based Adjuvant Therapy. JNCI Journal of the National Cancer Institute. 2011;103:863-75.

10. Zaanan A, Cuilliere-Dartigues P, Guilloux A, Parc Y, Louvet C, Gramont A de, et al. Impact of p53 expression and microsatellite instability on stage III colon cancer disease-free survival in patients treated by 5-fluorouracil and leucovorin with or without oxaliplatin. Annals of Oncology.

2010;21:772-80.

11. Tougeron D, Sickersen G, Lecomte T, Mouillet G, Trouilloud I, Coriat R, et al. Impact of adjuvant chemotherapy with 5-FU or FOLFOX in colon cancers with microsatellite instability: An AGEO multicenter study. Journal of Clinical Oncology. 2014;32:5s (suppl; abstr 3508). 
12. Collura A, Lagrange A, Svrcek M, Marisa L, Buhard O, Guilloux A, et al. Patients With Colorectal Tumors With Microsatellite Instability and Large Deletions in HSP110 T17 Have Improved Response to 5-Fluorouracil-Based Chemotherapy. Gastroenterology. 2014;146:401-11.

13. Dorard C, de Thonel A, Collura A, Marisa L, Svrcek M, Lagrange A, et al. Expression of a mutant HSP110 sensitizes colorectal cancer cells to chemotherapy and improves disease prognosis. Nature Medicine. 2011;17:1283-9.

14. Brueckl WM, Moesch C, Brabletz T, Koebnick C, Riedel C, Jung A, et al. Relationship between microsatellite instability, response and survival in palliative patients with colorectal cancer undergoing first-line chemotherapy. Anticancer Res. 2003;23:1773-7.

15. Des Guetz G, Mariani P, Cucherousset J, Benamoun M, Lagorce C, Sastre X, et al. Microsatellite instability and sensitivitiy to FOLFOX treatment in metastatic colorectal cancer. Anticancer research. 2007;27:2715-9.

16. Koopman M, Kortman GAM, Mekenkamp L, Ligtenberg MJL, Hoogerbrugge N, Antonini NF, et al. Deficient mismatch repair system in patients with sporadic advanced colorectal cancer. British Journal of Cancer. 2009;100:266-73.

17. Tran B, Kopetz S, Tie J, Gibbs P, Jiang Z-Q, Lieu CH, et al. Impact of BRAF mutation and microsatellite instability on the pattern of metastatic spread and prognosis in metastatic colorectal cancer. Cancer. 2011;117:4623-32.

18. $\bullet \bullet$ Venderbosch S, Nagtegaal ID, Maughan TS, Smith CG, Cheadle JP, Fisher D, et al. Mismatch Repair Status and BRAF Mutation Status in Metastatic Colorectal Cancer Patients: A Pooled Analysis of the CAIRO, CAIRO2, COIN, and FOCUS Studies. Clinical Cancer Research. 2014;20:5322-30. In metastatic colorectal cancer, both MMR deficiency and BRAFV6OOE mutation confer poor prognosis. The poor prognosis of $d M M R$ seens to be driven by BRAFV600E

19. Smith CG, Fisher D, Claes B, Maughan TS, Idziaszczyk S, Peuteman G, et al. Somatic Profiling of the Epidermal Growth Factor Receptor Pathway in Tumors from Patients with Advanced Colorectal Cancer Treated with Chemotherapy Cetuximab. Clinical Cancer Research. 2013;19:4104-13.

20. Donnard E, Asprino PF, Correa BR, Bettoni F, Koyama FC, Navarro FC, et al. Mutational analysis of genes coding for cell surface proteins in colorectal cancer cell lines reveal novel altered pathways, druggable mutations and mutated epitopes for targeted therapy. Oncotarget. 2014;5:9199.

21. Giannakis M, Shukla S, Mu J, Nishihara R, Yamauchi M, Sukawa Y, et al. Comprehensive molecular characterization of colorectal cancer reveals genomic predictors of immune cell infiltrates. Journal of Clinical Oncology. 2015;33 (suppl; abstr 3505).

22. Maby P, Tougeron D, Hamieh M, Mlecnik B, Kora H, Bindea G, et al. Correlation between density of CD8+ T cell infiltrates in microsatellite unstable colorectal cancers and frameshift mutations: a rationale for personalized immunotherapy. Cancer Research. Published Online First June $9,2015$.

23. Galon J, Costes A, Sanchez-Cabo F, Kirilovsky A, Mlecnik B, Lagorce-Pagès C, et al. Type, Density, and Location of Immune Cells Within Human Colorectal Tumors Predict Clinical Outcome. Science. 2006;313:1960-4.

24. Pagès F, Berger A, Camus M, Sanchez-Cabo F, Costes A, Molidor R, et al. Effector Memory T Cells, Early Metastasis, and Survival in Colorectal Cancer. New England Journal of Medicine. 2005;353:2654-66. 
25. Mlecnik B, Tosolini M, Charoentong P, Kirilovsky A, Bindea G, Berger A, et al. Biomolecular Network Reconstruction Identifies T-Cell Homing Factors Associated With Survival in Colorectal Cancer. Gastroenterology. 2010;138:1429-40.

26. Lal N, Beggs AD, Willcox BE, Middleton GW. An immunogenomic stratification of colorectal cancer: Implications for development of targeted immunotherapy. OncoImmunology. 2015;4:e976052.

27. $\bullet$ Llosa NJ, Cruise M, Tam A, Wicks EC, Hechenbleikner EM, Taube JM, et al. The Vigorous Immune Microenvironment of Microsatellite Instable Colon Cancer Is Balanced by Multiple CounterInhibitory Checkpoints. Cancer Discovery. 2015;5:43-51. Colorectal cancers with MMR deficiency specifically upregulate several immune checkpoints, unlike tumors with proficient MMR system.

28. Bodo S, Colas C, Buhard O, Collura A, Tinat J, Lavoine N, et al. Diagnosis of Constitutional Mismatch Repair-deficiency Syndrome Based on Microsatellite Instability and Lymphocyte Tolerance to Methylating Agents. Gastroenterology. Published Online First June 25, 2015

29. Rajagopalan H, Bardelli A, Lengauer C, Kinzler KW, Vogelstein B, Velculescu VE. Tumorigenesis: RAF/RAS oncogenes and mismatch-repair status. Nature. 2002;418:934-934.

30. Capper D, Voigt A, Bozukova G, Ahadova A, Kickingereder P, von Deimling A, et al. BRAF V600E-specific immunohistochemistry for the exclusion of Lynch syndrome in MSI-H colorectal cancer: BRAF V600E Immunohistochemistry in MSI-H Colorectal Cancer. International Journal of Cancer. 2013;133:1624-30.

31. Cremolini C, Bartolomeo MD, Amatu A, Antoniotti C, Moretto R, Berenato R, et al. BRAF codons 594 and 596 mutations identify a new molecular subtype of metastatic colorectal cancer at favorable prognosis. Ann Oncol. 2015; Published Online First July 7, 2015

32. Roth AD, Tejpar S, Delorenzi M, Yan P, Fiocca R, Klingbiel D, et al. Prognostic Role of KRAS and BRAF in Stage II and III Resected Colon Cancer: Results of the Translational Study on the PETACC-3, EORTC 40993, SAKK 60-00 Trial. Journal of Clinical Oncology. 2010;28:466-74.

33. André T, de Gramont A, Chibaudel B, Tijeras-Raballand A, Duval A, Hickish T, et al. MOSAIC study: Actualization of overall survival (OS) with 10 years follow up and evaluation of BRAF. By GERCOR and MOSAIC investigators. Annals of Oncology. 2014;25 suppl_4:iv167-209.

34. Taieb J, Tabernero J, Mini E, Subtil F, Folprecht G, Van Laethem J-L, et al. Oxaliplatin, fluorouracil, and leucovorin with or without cetuximab in patients with resected stage III colon cancer (PETACC-8): an open-label, randomised phase 3 trial. The Lancet Oncology. 2014;15:862-73.

35. Gonsalves WI, Mahoney MR, Sargent DJ, Nelson GD, Alberts SR, Sinicrope FA, et al. Patient and Tumor Characteristics and BRAF and KRAS Mutations in Colon Cancer, NCCTG/Alliance N0147. JNCI J Natl Cancer Inst. 2014;106:dju106.

36. French AJ, Sargent DJ, Burgart LJ, Foster NR, Kabat BF, Goldberg R, et al. Prognostic Significance of Defective Mismatch Repair and BRAF V600E in Patients with Colon Cancer. Clin Cancer Res. 2008;14:3408-15.

37. Gavin PG, Colangelo LH, Fumagalli D, Tanaka N, Remillard MY, Yothers G, et al. Mutation Profiling and Microsatellite Instability in Stage II and III Colon Cancer: An Assessment of Their Prognostic and Oxaliplatin Predictive Value. Clin Cancer Res. 2012;18:6531-41.

38. Taieb J, Le Malicot K, Penault-Llorca F, Bouche O, Shi Q, Thibodeau S, et al. Prognostic value of BRAF V600E and KRAS exon 2 mutations in microsatellite stable (MSS), stage III colon cancers 
(CC) from patients (pts) treated with adjuvant FOLFOX+/- cetuximab: A pooled analysis of 3934 pts from the PETACC8 and N0147 trials. Journal of Clinical Oncology. 2015;33 (suppl; abstr 3507).

39. Van Cutsem E, Köhne C-H, Láng I, Folprecht G, Nowacki MP, Cascinu S, et al. Cetuximab Plus Irinotecan, Fluorouracil, and Leucovorin As First-Line Treatment for Metastatic Colorectal Cancer: Updated Analysis of Overall Survival According to Tumor KRAS and BRAF Mutation Status. JCO. 2011;29:2011-9.

40. Douillard J-Y, Oliner KS, Siena S, Tabernero J, Burkes R, Barugel M, et al. PanitumumabFOLFOX4 Treatment and RAS Mutations in Colorectal Cancer. New England Journal of Medicine. 2013;369:1023-34.

41. Heinemann V, von Weikersthal LF, Decker T, Kiani A, Vehling-Kaiser U, Al-Batran S-E, et al. FOLFIRI plus cetuximab versus FOLFIRI plus bevacizumab as first-line treatment for patients with metastatic colorectal cancer (FIRE-3): a randomised, open-label, phase 3 trial. The Lancet Oncology. 2014;15:1065-75.

42. Stintzing S, Jung A, Rossius L, Modest DP, Fischer von Weikersthal L, Decker T, et al. Analysis of KRAS/NRAS and BRAF mutations in FIRE-3: A randomized phase III study of FOLFIRI plus cetuximab or bevacizumab as first-line treatment for wild-type (WT) KRAS (exon 2) metastatic colorectal cancer (mCRC) patients. European Cancer Congress. 2013;Abstract 17.

43. Peeters M, Price TJ, Cervantes Ruiperez A, Sobrero A, Ducreux M, André T, et al. Impact of baseline covariates and prior therapy on the efficacy of second-line panitumumab (pmab) + FOLFIRI vs FOLFIRI treatment. Annals of Oncology. 2014;25 (suppl_4):iv167-209.

44. André T, Blons H, Mabro M, Chibaudel B, Bachet J-B, Tournigand C, et al. Panitumumab combined with irinotecan for patients with KRAS wild-type metastatic colorectal cancer refractory to standard chemotherapy: a GERCOR efficacy, tolerance, and translational molecular study. Ann Oncol. $2013 ; 24: 412-9$.

45. De Roock W, Claes B, Bernasconi D, De Schutter J, Biesmans B, Fountzilas G, et al. Effects of KRAS, BRAF, NRAS, and PIK3CA mutations on the efficacy of cetuximab plus chemotherapy in chemotherapy-refractory metastatic colorectal cancer: a retrospective consortium analysis. The Lancet Oncology. 2010;11:753-62.

46. Seymour MT, Brown SR, Middleton G, Maughan T, Richman S, Gwyther S, et al. Panitumumab and irinotecan versus irinotecan alone for patients with KRAS wild-type, fluorouracil-resistant advanced colorectal cancer (PICCOLO): a prospectively stratified randomised trial. The lancet oncology. 2013;14:749-59.

47. Karapetis CS, Jonker D, Daneshmand M, Hanson JE, O'Callaghan CJ, Marginean C, et al. PIK3CA, BRAF, and PTEN Status and Benefit from Cetuximab in the Treatment of Advanced Colorectal Cancer-Results from NCIC CTG/AGITG CO.17. Clin Cancer Res. 2014;20:744-53.

48. $\bullet$ Rowland A, Dias MM, Wiese MD, Kichenadasse G, McKinnon RA, Karapetis CS, et al. Metaanalysis of BRAF mutation as a predictive biomarker of benefit from anti-EGFR monoclonal antibody therapy for RAS wild-type metastatic colorectal cancer. British Journal of Cancer. 2015;112:1888-94. In this meta-analysis, the hazard ratio for OS benefit with anti-EGFR monoclonal antibodies was 0.97 (95\% CI; 0.67-1.41) for BRAF ${ }^{V 600 E}$ tumors whereas the hazard ratio was 0.81 (95\% CI; 0.70-0.95) for $R A S^{W T} / B R A F^{W T} m C R C$. However the test of interaction was not statistically significant. Thus there is insufficient data to justify the exclusion of anti-EGFR therapies for patients with RAS $S^{W T} / B R A F^{V 600 E}$ $m C R C$ 
49. Seligmann J, Fisher D, Elliott F, Richman S, Butler R, Cheadle J, et al. Exploring the poor outcomes of BRAF mutant (BRAF mut) advanced colorectal cancer (aCRC): Analysis from 2,530 patients (pts) in randomized clinical trials (RCTs). Journal of Clinical Oncology. 2015;33 (suppl; abstr $3509)$.

50. Masi G, Loupakis F, Salvatore L, Fornaro L, Cremolini C, Cupini S, et al. Bevacizumab with FOLFOXIRI (irinotecan, oxaliplatin, fluorouracil, and folinate) as first-line treatment for metastatic colorectal cancer: a phase 2 trial. The Lancet Oncology. 2010;11:845-52.

51. Salvatore L, Loupakis F, Cremolini C, Schirripa M, Masi G, Antoniotti C, et al. FOLFOXIRI plus bevacizumab as first-line treatment of BRAF-mutant metastatic colorectal cancer patients. Journal of Clinical Oncology. 2012;30 suppl; abstr 3585.

52. Loupakis F, Cremolini C, Masi G, Lonardi S, Zagonel V, Salvatore L, et al. Initial Therapy with FOLFOXIRI and Bevacizumab for Metastatic Colorectal Cancer. New England Journal of Medicine. 2014;371:1609-18.

53. Loupakis F, Cremolini C, Lonardi S, Tomasello G, Ronzoni M, Zaniboni A, et al. Subgroup analyses in RAS mutant, BRAF mutant and all-wt mCRC pts treated with FOLFOXIRI plus bevacizumab (bev) or FOLFIRI plus bev in the TRIBE study. Journal of Clinical Oncology. 2014;32:5s (suppl; abstr 3519).

54. $\bullet$ Loupakis F, Cremolini C, Antoniotti C, Lonardi S, Ronzoni M, Zaniboni A, et al. FOLFOXIRI plus bevacizumab versus FOLFIRI plus bevacizumab as initial treatment for metastatic colorectal cancer (TRIBE study): Updated survival results and final molecular subgroups analyses. Journal of Clinical Oncology. 2015;33 (suppl; abstr 3510). Compared with FOLFIRI/bevacizumab, FOLFOXIRI/bevacizumab is associated with a non-significant increase of overall survival for patients with BRAFV600E metastatic colorectal cancers (19 months versus 10.7 months; $\mathrm{HR} 0.54,95 \% \mathrm{Cl}$ 0.24-1.20).

55. Chapman PB, Hauschild A, Robert C, Haanen JB, Ascierto P, Larkin J, et al. Improved survival with vemurafenib in melanoma with BRAF V600E mutation. New England Journal of Medicine. 2011;364:2507-16.

56. Hauschild A, Grob J-J, Demidov LV, Jouary T, Gutzmer R, Millward M, et al. Dabrafenib in BRAF-mutated metastatic melanoma: a multicentre, open-label, phase 3 randomised controlled trial. The Lancet. 2012;380:358-65.

57. Kopetz S, Desai J, Chan E, Hecht JR, O’Dwyer PJ, Lee RJ, et al. PLX4032 in metastatic colorectal cancer patients with mutant BRAF tumors. Journal of Clinical Oncology. 2010;28:15s (suppl; abstr $3534)$.

58. •• Prahallad A, Sun C, Huang S, Di Nicolantonio F, Salazar R, Zecchin D, et al.

Unresponsiveness of colon cancer to BRAF(V600E) inhibition through feedback activation of EGFR. Nature. 2012;483:100-3. In colorectal cancer, BRAFV600E inhibition induces a feedback activation of EGFR. The combination of vemurafenib with an anti-EGFR therapy or a MEK inhibitor may be an effective way to overpass BRAF inhibitor primary resistance in BRAF ${ }^{V 600 E}$ metastatic CRC.

59. Robert C, Karaszewska B, Schachter J, Rutkowski P, Mackiewicz A, Stroiakovski D, et al. Improved Overall Survival in Melanoma with Combined Dabrafenib and Trametinib. New England Journal of Medicine. 2015;372:30-9.

60. Long GV, Stroyakovskiy D, Gogas H, Levchenko E, de Braud F, Larkin J, et al. Dabrafenib and trametinib versus dabrafenib and placebo for Val600 BRAF-mutant melanoma: a multicentre, doubleblind, phase 3 randomised controlled trial. The Lancet. 2015;386:444-451 
61. Poulikakos PI, Zhang C, Bollag G, Shokat KM, Rosen N. RAF inhibitors transactivate RAF dimers and ERK signalling in cells with wild-type BRAF. Nature. 2010;464:427-30.

62. Hatzivassiliou G, Song K, Yen I, Brandhuber BJ, Anderson DJ, Alvarado R, et al. RAF inhibitors prime wild-type RAF to activate the MAPK pathway and enhance growth. Nature. 2010;464:431-5.

63. Corcoran RB, Atreya CE, Falchook GS, Infante JR, Hamid O, Messersmith WA, et al. Phase 1-2 trial of the BRAF inhibitor dabrafenib (D) plus MEK inhibitor trametinib (T) in BRAF V600 mutant colorectal cancer (CRC): Updated efficacy and biomarker analysis. Journal of Clinical Oncology. 2014;32:5s (suppl; abstr 3517).

64. Atreya C, Van Cutsem E, Bendell J, Andre T, Schellens J, Gordon M, et al. Updated efficacy of the MEK inhibitor trametinib (T), BRAF inhibitor dabrafenib (D), and anti-EGFR antibody panitumumab (P) in patients (pts) with BRAF V600E mutated (BRAFm) metastatic colorectal cancer (mCRC). Journal of Clinical Oncology. 2015;(suppl; abstr 103).

65. Yaeger R, Cercek A, O'Reilly EM, Reidy DL, Kemeny N, Wolinsky T, et al. Pilot Trial of Combined BRAF and EGFR Inhibition in BRAF-Mutant Metastatic Colorectal Cancer Patients. Clin Cancer Res. 2015;21:1313-20.

66. Hong D, Morris V, Osta B El, Fu S, Overman M, Piha-Paul S, et al. Phase Ib study of vemurafenib in combination with irinotecan and cetuximab in patients with BRAF-mutated metastatic colorectal cancer and advanced cancers. Journal of Clinical Oncology. 2015;33 (suppl; abstr 3511).

67. Colas C, Coulet F, Svrcek M, Collura A, Fléjou J-F, Duval A, et al. Lynch or not Lynch? Is that always a question? Adv. Cancer Res. 2012;113:121-66.

68. Shia J. Immunohistochemistry versus microsatellite instability testing for screening colorectal cancer patients at risk for hereditary nonpolyposis colorectal cancer syndrome. Part I. The utility of immunohistochemistry. J Mol Diagn. 2008;10:293-300.

69. Hampel H, Frankel WL, Martin E, Arnold M, Khanduja K, Kuebler P, et al. Screening for the Lynch syndrome (hereditary nonpolyposis colorectal cancer). New England Journal of Medicine. 2005;352:1851-60.

70. Boland CR, Thibodeau SN, Hamilton SR, Sidransky D, Eshleman JR, Burt RW, et al. A National Cancer Institute Workshop on Microsatellite Instability for Cancer Detection and Familial Predisposition: Development of International Criteria for the Determination of Microsatellite Instability in Colorectal Cancer. Cancer Research. 1998;58:5248-57.

71. Umar A, Boland CR, Terdiman JP, Syngal S, Chapelle A d. 1., Ruschoff J, et al. Revised Bethesda Guidelines for Hereditary Nonpolyposis Colorectal Cancer (Lynch Syndrome) and Microsatellite Instability. JNCI Journal of the National Cancer Institute. 2004;96:261-8.

72. Tabernero J, Chan E, Baselga J, Blay J-Y, Chau I, Hyman DM, et al. VE-BASKET, a Simon 2stage adaptive design, phase II, histology-independent study in nonmelanoma solid tumors harboring BRAF V600 mutations (V600m): Activity of vemurafenib (VEM) with or without cetuximab (CTX) in colorectal cancer (CRC). Journal of Clinical Oncology. 2014;32:5s (suppl; abstr 3518).

73. Gomez-Roca CA, Delord J, Robert C, Hidalgo M, von Moos R, Arance A, et al. Encorafenib (LGX818), an oral BRAF inhibitor, in patients (pts) with BRAF V600E metastatic colorectal cancer $(\mathrm{mCRC})$ : results of dose expansion in an open-label, phase 1 study. Annals of Oncology. 2014;25:iv182-3.

74. Drug Combo Beneficial in Colorectal Cancer. Cancer Discovery. 2015;5:102-102. 